\title{
Some inequalities in functional analysis, combinatorics, and probability theory
}

\author{
Chunrong Feng* Liangpan $\mathrm{Li}^{\dagger} \quad$ Jian Shen ${ }^{\ddagger}$
}

Submitted: Aug 21, 2009; Accepted: Mar 30, 2010; Published: Apr 5, 2010

Mathematics Subject Classification: 46C05, 05A20, 60C05, 11 T99

\begin{abstract}
The main purpose of this paper is to show that many inequalities in functional analysis, probability theory and combinatorics are immediate corollaries of the best approximation theorem in inner product spaces. Besides, as applications of the de Caen-Selberg inequality, the finite field Kakeya and Nikodym problems are also studied.
\end{abstract}

Keywords: inner product space, orthogonal projection, Kakeya set, Nikodym set

\section{Brief Introduction}

Let $(H,<\cdot, \cdot>)$ be an inner product space over $\mathbb{R}$ throughout. Given $x \in H$ and a finite dimensional subspace $M$, denote by $x_{M}$ the orthogonal projection of $x$ onto $M$. It is geometrically evident that (we always assume $\frac{0}{0}=0$ in this paper)

$$
\|x\|^{2} \geqslant\left\|x_{M}\right\|^{2}=\max _{y \in M} \frac{<x_{M}, y>^{2}}{\|y\|^{2}}=\max _{y \in M} \frac{<x, y>^{2}}{\|y\|^{2}} .
$$

Particularly, if $M=\operatorname{span}\left\{y_{i}\right\}_{i=1}^{n}$ for some given set of elements $y_{1}, \ldots, y_{n}$, then

$$
\|x\|^{2} \geqslant \max _{\left(\alpha_{1}, \ldots, \alpha_{n}\right) \in \mathbb{R}^{n}} \frac{<x, \sum_{i=1}^{n} \alpha_{i} y_{i}>^{2}}{\left\|\sum_{i=1}^{n} \alpha_{i} y_{i}\right\|^{2}} .
$$

${ }^{*}$ Department of Mathematics, Shanghai Jiao Tong University, Shanghai 200240, China \& Department of Mathematical Sciences, Loughborough University, Leics, LE11 3TU, UK. E-mail: fcr@sjtu.edu.cn. Research was supported by the Mathematical Tianyuan Foundation of China (No. 10826090).

${ }^{\dagger}$ Department of Mathematics, Shanghai Jiao Tong University, Shanghai 200240, China. E-mail: liliangpan@yahoo.com.cn. Research was supported by the Mathematical Tianyuan Foundation of China (No. 10826088).

${ }^{\ddagger}$ Department of Mathematics, Texas State University, San Marcos, TX 78666, USA. E-mail: js48@txstate.edu. Research was supported by NSF (CNS 0835834) and Texas Higher Education Coordinating Board (ARP 003615-0039-2007). 
The main purpose of this paper is to show that many inequalities in functional analysis, probability theory and combinatorics are immediate corollaries of (2). For the sake of completeness we determine the unique orthogonal projection $x_{M}$ (many authors of textbooks on functional analysis only dealt the case when $\left\{y_{i}\right\}_{i=1}^{n}$ are linear independent). Write $x_{M}=\sum_{i=1}^{n} \beta_{i} y_{i}$ for some $\left(\beta_{1}, \ldots, \beta_{n}\right) \in \mathbb{R}^{n}$. Since the smooth function

$$
\Psi\left(\alpha_{1}, \ldots, \alpha_{n}\right) \doteq\left\|x-\sum_{i=1}^{n} \alpha_{i} y_{i}\right\|^{2}=\|x\|^{2}-2 \sum_{i=1}^{n} \alpha_{i}<x, y_{i}>+\sum_{i=1}^{n} \sum_{j=1}^{n} \alpha_{i} \alpha_{j}<y_{i}, y_{j}>
$$

attains its minimum $d(x, M)^{2}$ at $\left(\beta_{1}, \ldots, \beta_{n}\right)$,

$$
\frac{\partial \Psi}{\partial \alpha_{i}}\left(\beta_{1}, \ldots, \beta_{n}\right)=0 \quad(i=1,2, \ldots, n) .
$$

Equivalently,

$$
\left(\begin{array}{cccc}
<y_{1}, y_{1}> & <y_{1}, y_{2}> & \cdots & <y_{1}, y_{n}> \\
<y_{2}, y_{1}> & <y_{2}, y_{2}> & \cdots & <y_{2}, y_{n}> \\
\vdots & \vdots & \ddots & \vdots \\
<y_{n}, y_{1}> & <y_{n}, y_{2}> & \cdots & <y_{n}, y_{n}>
\end{array}\right)\left(\begin{array}{c}
\beta_{1} \\
\beta_{2} \\
\vdots \\
\beta_{n}
\end{array}\right)=\left(\begin{array}{c}
<x, y_{1}> \\
<x, y_{2}> \\
\vdots \\
<x, y_{n}>
\end{array}\right)
$$

If $\left(\gamma_{1}, \ldots, \gamma_{n}\right) \in \mathbb{R}^{n}$ is another solution to $(3)$, then

$$
\begin{aligned}
\left\|\sum_{i=1}^{n}\left(\beta_{i}-\gamma_{i}\right) y_{i}\right\|^{2} & =\left(\beta_{1}-\gamma_{1}, \cdots, \beta_{n}-\gamma_{n}\right)\left(<y_{i}, y_{j}>\right)_{n \times n}\left(\begin{array}{c}
\beta_{1}-\gamma_{1} \\
\vdots \\
\beta_{n}-\gamma_{n}
\end{array}\right) \\
& =\left(\beta_{1}-\gamma_{1}, \cdots, \beta_{n}-\gamma_{n}\right)\left(\begin{array}{c}
0 \\
\vdots \\
0
\end{array}\right)=0 .
\end{aligned}
$$

Consequently $x_{M}=\sum_{i=1}^{n} \beta_{i} y_{i}=\sum_{i=1}^{n} \gamma_{i} y_{i}$.

Among many inequalities will be discussed later, we show particular interest in the de Caen-Selberg inequality $[1,2]$ :

$$
\left|\bigcup_{i=1}^{n} A_{i}\right| \geqslant \sum_{i=1}^{n} \frac{\left|A_{i}\right|^{2}}{\sum_{j=1}^{n}\left|A_{i} \cap A_{j}\right|}
$$

where $\left\{A_{i}\right\}_{i=1}^{n}$ are finite sets. In Section 5 we will present some applications of the de Caen-Selberg inequality to the study of the finite field Kakeya and Nikodym problems in classical analysis. 


\section{Inequalities in Functional Analysis}

\subsection{Known inequalities}

For any $\left(\alpha_{1}, \ldots, \alpha_{n}\right) \in \mathbb{R}^{n}$, by $(2)$ and the Cauchy-Schwarz inequality $\left(\left|\alpha_{i} \alpha_{j}\right| \leqslant \frac{\alpha_{i}^{2}+\alpha_{j}^{2}}{2}\right)$ one obtains the Pečarić inequality [13]

$$
\|x\|^{2} \geqslant \frac{\left(\sum_{i=1}^{n} \alpha_{i}<x, y_{i}>\right)^{2}}{\sum_{i=1}^{n} \sum_{j=1}^{n} \alpha_{i}^{2}\left|<y_{i}, y_{j}>\right|} .
$$

(The following arguments are standard [13]) Substituting $\alpha_{i}=\frac{<x, y_{i}>}{\sum_{k=1}^{n}\left|<y_{i}, y_{k}>\right|}$ into (5) yields the Selberg inequality [1]

$$
\|x\|^{2} \geqslant \sum_{i=1}^{n} \frac{<x, y_{i}>^{2}}{\sum_{j=1}^{n}\left|<y_{i}, y_{j}\right\rangle \mid}
$$

Substituting $\alpha_{i}=\operatorname{sgn}\left(<x, y_{i}>\right)$ into (5) or applying the Cauchy-Schwarz inequality from (6) yields the Heilbronn inequality [10]

$$
\|x\|^{2} \geqslant \frac{\left(\sum_{i=1}^{n}\left|<x, y_{i}>\right|\right)^{2}}{\sum_{i=1}^{n} \sum_{j=1}^{n}\left|<y_{i}, y_{j}>\right|} .
$$

The Selberg inequality (6) is certainly stronger than the Bombieri inequality [1]

$$
\|x\|^{2} \geqslant \frac{\left.\sum_{i=1}^{n}<x, y_{i}\right\rangle^{2}}{\max _{1 \leqslant i \leqslant n} \sum_{j=1}^{n}\left|<y_{i}, y_{j}\right\rangle \mid} .
$$

If $\left\{y_{i}\right\}_{i=1}^{n}$ are orthogonal, then the Selberg inequality (6) turns out to be the classical Bessel inequality

$$
\|x\|^{2} \geqslant \sum_{i=1}^{n} \frac{<x, y_{i}>^{2}}{<y_{i}, y_{i}>}
$$

Substituting $\alpha_{i}=1$ into (2) yields the Chung-Erdős inequality [3]

$$
\|x\|^{2} \geqslant \frac{\left(\sum_{i=1}^{n}<x, y_{i}>\right)^{2}}{\sum_{i=1}^{n} \sum_{j=1}^{n}<y_{i}, y_{j}>} .
$$


In a partial summary,

$$
\text { (2) } \succ(5) \succ(6) \succ(7) \text {, }
$$

where $(\bullet) \succ(\bullet \bullet)$ means Estimate $(\bullet)$ is stronger than Estimate $(\bullet \bullet)$.

\section{From Functional Analysis to Combinatorics}

\subsection{Immediate corollaries}

In this section we always choose $H=l^{2}$. Let $A, B$ be finite subsets of $\mathbb{N}$ and $\chi_{A}, \chi_{B}$ be the corresponding indictor functions. Then

$$
<\chi_{A}, \chi_{B}>=|A \cap B|,
$$

and $\chi_{A}, \chi_{B}$ are orthogonal means $A, B$ are disjoint sets. Given finite subsets $\left\{A_{i}\right\}_{i=1}^{n}$ of $\mathbb{N}$, define $y_{i}=\chi_{A_{i}}(i \in[n])$ and $x=\chi_{\cup_{i} A_{i}}$. Then $\left.<x, y_{i}\right\rangle=\left|\left(\cup_{j} A_{j}\right) \cap A_{i}\right|=\left|A_{i}\right|$. By $(2)$ and (3), we obtain

\section{Theorem 3.1.}

$$
\left|\bigcup_{i=1}^{n} A_{i}\right| \geqslant \max _{\left(\alpha_{1}, \ldots, \alpha_{n}\right) \in \mathbb{R}^{n}} \frac{\left(\sum_{i=1}^{n} \alpha_{i}\left|A_{i}\right|\right)^{2}}{\sum_{i=1}^{n} \sum_{j=1}^{n} \alpha_{i} \alpha_{j}\left|A_{i} \cap A_{j}\right|}=\sum_{i=1}^{n} \sum_{j=1}^{n} \beta_{i} \beta_{j}\left|A_{i} \cap A_{j}\right|,
$$

where $\left(\beta_{1}, \ldots, \beta_{n}\right) \in \mathbb{R}^{n}$ is any solution to

$$
\left(\begin{array}{cccc}
\left|A_{1} \cap A_{1}\right| & \left|A_{1} \cap A_{2}\right| & \cdots & \left|A_{1} \cap A_{n}\right| \\
\left|A_{2} \cap A_{1}\right| & \left|A_{2} \cap A_{2}\right| & \cdots & \left|A_{2} \cap A_{n}\right| \\
\vdots & \vdots & \ddots & \vdots \\
\left|A_{n} \cap A_{1}\right| & \left|A_{n} \cap A_{2}\right| & \cdots & \left|A_{n} \cap A_{n}\right|
\end{array}\right)\left(\begin{array}{c}
\beta_{1} \\
\beta_{2} \\
\vdots \\
\beta_{n}
\end{array}\right)=\left(\begin{array}{c}
\left|A_{1}\right| \\
\left|A_{2}\right| \\
\vdots \\
\left|A_{n}\right|
\end{array}\right)
$$

Note in this context the Selberg inequality (6) turns out to be the de Caen inequality (4) and the Bessel inequality (9) turns out to be a trivial equality. Also note that

$$
\sup _{\alpha_{i}>0} \frac{\left(\sum_{i=1}^{n} \alpha_{i}\left|A_{i}\right|\right)^{2}}{\sum_{i=1}^{n} \sum_{j=1}^{n} \alpha_{i} \alpha_{j}\left|A_{i} \cap A_{j}\right|}=\sup _{\alpha_{i}>0} \frac{\left(\sum_{i=1}^{n} \alpha_{i}\left|A_{i}\right|\right)^{2}}{\sum_{i=1}^{n} \sum_{j=1}^{n} \alpha_{i}^{2}\left|A_{i} \cap A_{j}\right|}=\sup _{\alpha_{i}>0} \sum_{i=1}^{n} \frac{\alpha_{i}\left|A_{i}\right|^{2}}{\sum_{j=1}^{n} \alpha_{j}\left|A_{i} \cap A_{j}\right|} .
$$




\subsection{A slightly different variant}

In this subsection, we provide a slightly different variant of (12).

Theorem 3.2. The following matrix equation always has a solution

$$
\left(\frac{\left|A_{i} \cap A_{j}\right|}{\left|A_{i}\right|\left|A_{j}\right|}\right)_{n \times n}\left(\begin{array}{c}
q_{1} \\
q_{2} \\
\vdots \\
q_{n}
\end{array}\right)=\left(\begin{array}{c}
1 \\
1 \\
\vdots \\
1
\end{array}\right)
$$

any solution to (13) satisfies

$$
\sum_{i=1}^{n} q_{i}=\max _{\left(\alpha_{1}, \ldots, \alpha_{n}\right) \in \mathbb{R}^{n}} \frac{\left(\sum_{i=1}^{n} \alpha_{i}\left|A_{i}\right|\right)^{2}}{\sum_{i=1}^{n} \sum_{j=1}^{n} \alpha_{i} \alpha_{j}\left|A_{i} \cap A_{j}\right|} .
$$

Proof. Write $P=\left(\frac{\left|A_{i} \cap A_{j}\right|}{\left|A_{i}\right|\left|A_{j}\right|}\right)_{n \times n}, Q=\left(\left|A_{i} \cap A_{j}\right|\right)_{n \times n}$ and $R=\operatorname{diag}\left(1 /\left|A_{1}\right|, \ldots, 1 /\left|A_{n}\right|\right)$. Obviously, $P=R Q R, Q=R^{-1} P R^{-1}$. Let $\left(\beta_{1}, \ldots, \beta_{n}\right) \in \mathbb{R}^{n}$ be a solution to (12). Then

$$
P\left(\begin{array}{c}
\beta_{1}\left|A_{1}\right| \\
\beta_{2}\left|A_{2}\right| \\
\vdots \\
\beta_{n}\left|A_{n}\right|
\end{array}\right)=R R^{-1} P R^{-1}\left(\begin{array}{c}
\beta_{1} \\
\beta_{2} \\
\vdots \\
\beta_{n}
\end{array}\right)=R Q\left(\begin{array}{c}
\beta_{1} \\
\beta_{2} \\
\vdots \\
\beta_{n}
\end{array}\right)=R\left(\begin{array}{c}
\left|A_{1}\right| \\
\left|A_{2}\right| \\
\vdots \\
\left|A_{n}\right|
\end{array}\right)=\left(\begin{array}{c}
1 \\
1 \\
\vdots \\
1
\end{array}\right) .
$$

This solves the existence. Suppose $\left(q_{1}, q_{2}, \cdots, q_{n}\right)^{T}$ is a solution to (13), that is,

$$
\operatorname{RQR}\left(\begin{array}{c}
q_{1} \\
q_{2} \\
\vdots \\
q_{n}
\end{array}\right)=\left(\begin{array}{c}
1 \\
1 \\
\vdots \\
1
\end{array}\right) \Leftrightarrow Q\left(\begin{array}{c}
q_{1} /\left|A_{1}\right| \\
q_{2} /\left|A_{2}\right| \\
\vdots \\
q_{n} /\left|A_{n}\right|
\end{array}\right)=\left(\begin{array}{c}
\left|A_{1}\right| \\
\left|A_{2}\right| \\
\vdots \\
\left|A_{n}\right|
\end{array}\right)
$$

By (11), (12) and (13),

$$
\begin{gathered}
\max _{\left(\alpha_{1}, \ldots, \alpha_{n}\right) \in \mathbb{R}^{n}} \frac{\left(\sum_{i=1}^{n} \alpha_{i}\left|A_{i}\right|\right)^{2}}{\sum_{i=1}^{n} \sum_{j=1}^{n} \alpha_{i} \alpha_{j}\left|A_{i} \cap A_{j}\right|}=\sum_{i=1}^{n} \sum_{j=1}^{n} \frac{q_{i}}{\left|A_{i}\right|} \cdot \frac{q_{j}}{\left|A_{j}\right|} \cdot\left|A_{i} \cap A_{j}\right| \\
=\left(q_{1}, q_{2}, \cdots, q_{n}\right) P\left(\begin{array}{c}
q_{1} \\
q_{2} \\
\vdots \\
q_{n}
\end{array}\right)=\left(q_{1}, q_{2}, \cdots, q_{n}\right)\left(\begin{array}{c}
1 \\
1 \\
\vdots \\
1
\end{array}\right)=\sum_{i=1}^{n} q_{i} .
\end{gathered}
$$

So we get (14). This concludes the whole proof. 


\subsection{A combinatorial proof}

In this subsection, we provide a combinatorial proof for the inequality in (11) to help understand the equality case. To achieve the goal we need only prove

$$
\left|\bigcup_{i=1}^{n} A_{i}\right| \geqslant \frac{\left(\sum_{i=1}^{n} \alpha_{i}\left|A_{i}\right|\right)^{2}}{\sum_{i=1}^{n} \sum_{j=1}^{n} \alpha_{i} \alpha_{j}\left|A_{i} \cap A_{j}\right|} .
$$

holds for all integral weights $\alpha_{i} \in \mathbb{Z}$ such that $\sum_{i=1}^{n} \alpha_{i}\left|A_{i}\right|>0$. Suppose this is the case. Let $U=\cup_{i=1}^{n} A_{i}$ and $\chi_{i}$ be the indicator function of $A_{i}$. Define $f(x)=\sum_{i=1}^{n} \alpha_{i} \chi_{i}(x)$ and for all $k \in \mathbb{Z}$,

$$
U^{k} \doteq\{x \in U: f(x)=k\}, \quad A_{i}^{k} \doteq A_{i} \cap U^{k} .
$$

Obviously, $f=\sum_{k \in \mathbb{Z}} k \chi_{U^{k}}$. Note

$$
\sum_{i=1}^{n} \alpha_{i}\left|A_{i}^{k}\right|=\sum_{i=1}^{n} \alpha_{i} \int_{U} \chi_{A_{i} \cap U^{k}}=\sum_{i=1}^{n} \alpha_{i} \int_{U} \chi_{i} \cdot \chi_{U^{k}}=\int_{U} f \cdot \chi_{U^{k}}=k \cdot\left|U^{k}\right|,
$$

and

$$
\sum_{k \in \mathbb{Z}} k\left|A_{i}^{k}\right|=\sum_{k \in \mathbb{Z}} k \int_{U} \chi_{i} \cdot \chi_{U^{k}}=\int_{A_{i}} \sum_{k \in \mathbb{Z}} k_{U^{k}}=\int_{A_{i}} \sum_{j=1}^{n} \alpha_{j} \chi_{j}=\sum_{j=1}^{n} \alpha_{j}\left|A_{i} \cap A_{j}\right|,
$$

here the integration means $\int_{U} g=\sum_{x \in U} g(x)$. By (15),

$$
|U|=\sum_{k \in \mathbb{Z}}\left|U^{k}\right| \geqslant \sum_{k \neq 0} \frac{\sum_{i=1}^{n} \alpha_{i}\left|X_{i}^{k}\right|}{k} .
$$

Now we need an inequality: for all $r, s>0$ one has

$$
\frac{1}{s} \geqslant \frac{2}{r}-\frac{s}{r^{2}} \quad\left(\Leftrightarrow\left(\frac{1}{s}-\frac{1}{r}\right)^{2} \geqslant 0\right)
$$

By (15) again, $\sum_{i=1}^{n} \alpha_{i}\left|A_{i}^{k}\right|$ and $k$ have the same sign, and consequently for $r>0$,

$$
\begin{aligned}
& \frac{\sum_{i=1}^{n} \alpha_{i}\left|A_{i}^{k}\right|}{k} \geqslant\left\{\begin{aligned}
\frac{2}{r} \sum_{i=1}^{n} \alpha_{i}\left|A_{i}^{k}\right|-\frac{k}{r^{2}} \sum_{i=1}^{n} \alpha_{i}\left|A_{i}^{k}\right| & \text { if } k>0 \\
-\frac{2}{r} \sum_{i=1}^{n} \alpha_{i}\left|A_{i}^{k}\right|-\frac{k}{r^{2}} \sum_{i=1}^{n} \alpha_{i}\left|A_{i}^{k}\right| & \text { if } k<0
\end{aligned}\right. \\
& \geqslant \frac{2}{r} \sum_{i=1}^{n} \alpha_{i}\left|A_{i}^{k}\right|-\frac{k}{r^{2}} \sum_{i=1}^{n} \alpha_{i}\left|A_{i}^{k}\right| \text { if } k \neq 0 .
\end{aligned}
$$

Recall that $\frac{2}{r} \sum_{i=1}^{n} \alpha_{i}\left|A_{i}^{k}\right|-\frac{k}{r^{2}} \sum_{i=1}^{n} \alpha_{i}\left|A_{i}^{k}\right|=0$ when $k=0$. By (16),

$$
|U| \geqslant \sum_{k \in \mathbb{Z}}\left(\frac{2}{r} \sum_{i=1}^{n} \alpha_{i}\left|A_{i}^{k}\right|-\frac{k}{r^{2}} \sum_{i=1}^{n} \alpha_{i}\left|A_{i}^{k}\right|\right)=\frac{2}{r} \sum_{i=1}^{n} \alpha_{i}\left|A_{i}\right|-\frac{1}{r^{2}} \sum_{i=1}^{n} \sum_{j=1}^{n} \alpha_{i} \alpha_{j}\left|A_{i} \cap A_{j}\right| \doteq W(r) .
$$


Finally,

$$
|U| \geqslant \max _{r>0} W(r)=W\left(r^{*}\right)=\frac{\left(\sum_{i=1}^{n} \alpha_{i}\left|A_{i}\right|\right)^{2}}{\sum_{i=1}^{n} \sum_{j=1}^{n} \alpha_{i} \alpha_{j}\left|A_{i} \cap A_{j}\right|}
$$

where $r^{*}=\left(\sum_{i=1}^{n} \alpha_{i}\left|A_{i}\right|\right) /\left(\sum_{i=1}^{n} \sum_{j=1}^{n} \alpha_{i} \alpha_{j}\left|A_{i} \cap A_{j}\right|\right)$. This concludes the whole proof. A byproduct of this proof is the following characterization of the equality case:

$$
\left|\bigcup_{i=1}^{n} A_{i}\right|=\left.\frac{\left(\sum_{i=1}^{n} \alpha_{i}\left|A_{i}\right|\right)^{2}}{\sum_{i=1}^{n} \sum_{j=1}^{n} \alpha_{i} \alpha_{j}\left|A_{i} \cap A_{j}\right|} \Leftrightarrow \sum_{i=1}^{n} \alpha_{i} \chi_{i}(x)\right|_{\bigcup_{i=1}^{n} A_{i}} \text { is a non-zero constant function. }
$$

\section{From Functional Analysis to Probability Theory}

\subsection{Finitely many events}

In this section we choose $H$ to be the $L^{2}$ space of the given probability space $(\Omega, \mathcal{F}, P)$. Let $E, F$ be two events and $\chi_{E}, \chi_{F}$ be the corresponding indicator functions. It is well-known that Hilbert space theory and probability theory are intimately connected by

$$
<\chi_{E}, \chi_{F}>=P(E \cap F) .
$$

Note $\chi_{E}, \chi_{F}$ are orthogonal means $E, F$ are disjoint. Given events $\left\{E_{i}\right\}_{i=1}^{n}$, define $y_{i}=$ $\chi_{E_{i}}(i \in[n])$ and $x=\chi_{\cup_{i} E_{i}}$. By (2) and (3), we extend the Gallot-Kounias inequality $[9,11]$ to its full generality in the following form.

Theorem 4.1 (Gallot-Kounias).

$$
P\left(\bigcup_{i=1}^{n} E_{i}\right) \geqslant \max _{\left(\alpha_{1}, \ldots, \alpha_{n}\right) \in \mathbb{R}^{n}} \frac{\left(\sum_{i=1}^{n} \alpha_{i} P\left(E_{i}\right)\right)^{2}}{\sum_{i=1}^{n} \sum_{j=1}^{n} \alpha_{i} \alpha_{j} P\left(E_{i} \cap E_{j}\right)}=\sum_{i=1}^{n} \sum_{j=1}^{n} \gamma_{i} \gamma_{j} P\left(E_{i} \cap E_{j}\right),
$$

where $\left(\gamma_{1}, \ldots, \gamma_{n}\right) \in \mathbb{R}^{n}$ is any solution to

$$
\left(\begin{array}{cccc}
P\left(E_{1} \cap E_{1}\right) & P\left(E_{1} \cap E_{2}\right) & \cdots & P\left(E_{1} \cap E_{n}\right) \\
P\left(E_{2} \cap E_{1}\right) & P\left(E_{2} \cap E_{2}\right) & \cdots & P\left(E_{2} \cap E_{n}\right) \\
\vdots & \vdots & \ddots & \vdots \\
P\left(E_{n} \cap E_{1}\right) & P\left(E_{n} \cap E_{2}\right) & \cdots & P\left(E_{n} \cap E_{n}\right)
\end{array}\right)\left(\begin{array}{c}
\gamma_{1} \\
\gamma_{2} \\
\vdots \\
\gamma_{n}
\end{array}\right)=\left(\begin{array}{c}
P\left(E_{1}\right) \\
P\left(E_{2}\right) \\
\vdots \\
P\left(E_{n}\right)
\end{array}\right)
$$


To the authors' knowledge, it seems that the Gallot-Kounias inequality, being discovered 40 years ago, was almost forgotten by Mathematicians. Gallot and Kounias originally expressed their results in terms of generalized inverse of matrices, and this may prevent their results from being appreciated by others. So we restate their results in a more natural way in Theorem 4.1. Note in this context (10) turns out to be the original Chung-Erdös inequality [3]

$$
P\left(\bigcup_{i=1}^{n} E_{i}\right) \geqslant \frac{\left(\sum_{i=1}^{n} P\left(E_{i}\right)\right)^{2}}{\sum_{i=1}^{n} \sum_{j=1}^{n} P\left(E_{i} \cap E_{j}\right)}
$$

and the Bessel inequality (9) turns out to be a trivial equality. Also note that

$$
\sup _{\alpha_{i}>0} \frac{\left(\sum_{i=1}^{n} \alpha_{i} P\left(E_{i}\right)\right)^{2}}{\sum_{i=1}^{n} \sum_{j=1}^{n} \alpha_{i} \alpha_{j} P\left(E_{i} \cap E_{j}\right)}=\sup _{\alpha_{i}>0} \frac{\left(\sum_{i=1}^{n} \alpha_{i} P\left(E_{i}\right)\right)^{2}}{\sum_{i=1}^{n} \sum_{j=1}^{n} \alpha_{i}^{2} P\left(E_{i} \cap E_{j}\right)}=\sup _{\alpha_{i}>0} \sum_{i=1}^{n} \frac{\alpha_{i} P\left(E_{i}\right)^{2}}{\sum_{j=1}^{n} \alpha_{j} P\left(E_{i} \cap E_{j}\right)} .
$$

Similar to Theorem 3.2 one can establish the following theorem.

Theorem 4.2. The following matrix equation always has a solution

$$
\left(\frac{P\left(E_{i} \cap E_{j}\right)}{P\left(E_{i}\right) P\left(E_{j}\right)}\right)_{n \times n}\left(\begin{array}{c}
q_{1} \\
q_{2} \\
\vdots \\
q_{n}
\end{array}\right)=\left(\begin{array}{c}
1 \\
1 \\
\vdots \\
1
\end{array}\right)
$$

any solution to (20) satisfies

$$
\sum_{i=1}^{n} q_{i}=\max _{\left(\alpha_{1}, \ldots, \alpha_{n}\right) \in \mathbb{R}^{n}} \frac{\left(\sum_{i=1}^{n} \alpha_{i} P\left(E_{i}\right)\right)^{2}}{\sum_{i=1}^{n} \sum_{j=1}^{n} \alpha_{i} \alpha_{j} P\left(E_{i} \cap E_{j}\right)}
$$

\subsection{Borel-Cantelli lemma}

Let $\left\{E_{i}\right\}_{i=1}^{\infty}$ be infinitely many events on the probability space $(\Omega, \mathcal{F}, P)$. The BorelCantelli lemma states that: (a) if $\sum_{i=1}^{\infty} P\left(E_{i}\right)<\infty$, then $P\left(\lim \sup E_{i}\right)=0$; (b) if $\sum_{i=1}^{\infty} P\left(E_{i}\right)=\infty$ and $\left\{E_{i}\right\}_{i=1}^{\infty}$ are mutually independent, then $P\left(\lim \sup E_{i}\right)=1$. Here $\limsup E_{i}=\cap_{i=1}^{\infty} \cup_{k=i}^{\infty} E_{k}$. The Borel-Cantelli lemma played an exceptionally important role in probability theory, and many investigations were devoted to the second part of the Borel-Cantelli lemma in the attempt to weaken the independence condition on $\left\{E_{i}\right\}_{i=1}^{\infty}$. 
Towards this question, Erdős and Rényi $[6,14]$ obtained a nice result closely related to (19): if $\sum_{i=1}^{\infty} P\left(E_{i}\right)=\infty$, then

$$
P\left(\limsup E_{i}\right) \geqslant \limsup _{n \rightarrow \infty} \frac{\left(\sum_{k=1}^{n} P\left(E_{k}\right)\right)^{2}}{\sum_{i=1}^{n} \sum_{j=1}^{n} P\left(E_{i} \cap E_{j}\right)} .
$$

Recently, by carefully studying the effect of the denominator in the right hand of (22), the authors [8] established a weighted version of the Erdős-Rényi theorem which states:

Theorem 4.3 (Feng-Li-Shen). If $\sum_{i=1}^{\infty} \alpha_{i} P\left(E_{i}\right)=\infty$, then

$$
P\left(\limsup E_{i}\right) \geqslant \limsup _{n \rightarrow \infty} \frac{\left(\sum_{k=1}^{n} \alpha_{k} P\left(E_{k}\right)\right)^{2}}{\sum_{i=1}^{n} \sum_{j=1}^{n} \alpha_{i} \alpha_{j} P\left(E_{i} \cap E_{j}\right)} .
$$

\section{Applications of the de Caen-Selberg Inequality}

\subsection{The finite field Kakeya set}

Let $\mathbb{F}_{q}$ denote a finite field of $q$ elements. Define a set $K \subset \mathbb{F}_{q}^{n}$ to be Kakeya if it contains a translate of any given line. The finite field Kakeya problem, posed by Wolff in his influential survey [17], conjectured that $|K| \geqslant C_{n} q^{n}$ holds for some constant $C_{n}$. Recently, using the polynomial method in algebraic extremal combinatorics, Dvir [4] completely confirmed this conjecture by proving

$$
|K| \geqslant\left(\begin{array}{c}
n+q-1 \\
n
\end{array}\right) .
$$

If $n=2$, it is well-known that (24) is sharp [7] and can be established by a simple counting argument [15]. For $n \geqslant 3$, see [16] for further improvement.

Similarly, we say a subset $E \subset \mathbb{F}_{q}^{n}$ is an $(n, k)$-set if it contains a translate of any given $k$-plane. Ellenberg, Oberlin and Tao [5] proved that if $2 \leqslant k<n$, then

$$
|E| \geqslant q^{n}-\left(\begin{array}{l}
n \\
2
\end{array}\right) q^{n-k+1}+o\left(q^{n-k+1}\right) \quad(q \rightarrow \infty) .
$$

Using the de Caen-Selberg inequality we can slightly improve (25) when $k=n-1 \geqslant 2$.

Theorem 5.1. Any $(n, n-1)$-set $E \subset \mathbb{F}_{q}^{n}(n \geqslant 3)$ satisfies

$$
|E| \geqslant q^{n}-q^{2}+o\left(q^{2}\right) \quad(q \rightarrow \infty),
$$

where $\mathbb{F}_{q}$ denotes a finite field of $q$ elements. 
Proof. Since the total number $s$ of $(n-1)$-dimensional hyperplanes passing through the origin equals the total number of lines passing through the origin,

$$
s=\frac{q^{n}-1}{q-1} .
$$

Let $\left\{P_{i}\right\}_{i=1}^{s}$ be such hyperplanes. By the de Caen-Selberg inequality (4),

$$
\begin{aligned}
|E| & \geqslant \sum_{i=1}^{s} \frac{\left|P_{i}\right|^{2}}{\sum_{j=1}^{s}\left|P_{i} \cap P_{j}\right|} \geqslant \frac{s \cdot q^{2 n-2}}{q^{n-1}+(s-1) q^{n-2}} \\
& =\frac{s \cdot q^{2 n-2}+q^{n}\left(q^{n-1}-q^{n-2}\right)-q^{n}\left(q^{n-1}-q^{n-2}\right)}{\left(q^{n-1}-q^{n-2}\right)+s \cdot q^{n-2}} \\
& =q^{n}-\frac{q^{n}\left(q^{n-1}-q^{n-2}\right)}{q^{n-1}+(s-1) q^{n-2}} \\
& =q^{n}-q^{2}+o\left(q^{2}\right) \quad(q \rightarrow \infty) .
\end{aligned}
$$

\subsection{The finite field Nikodym set}

Define a set $B \subset \mathbb{F}_{q}^{n}$ to be Nikodym if for each $z \in B^{c}$ there exists a line $L_{z}$ passing through $z$ such that $L_{z} \backslash\{z\} \subset B$. Obviously, all such lines $\left\{L_{z}\right\}_{z \in B^{c}}$ are different from each other. Similar to (24) Li [12] proved (i)

$$
|B| \geqslant\left(\begin{array}{c}
n+q-2 \\
n
\end{array}\right)
$$

(ii) any two-dimensional Nikodym set $B \subset \mathbb{F}_{q}^{2}$ satisfies

$$
|B| \geqslant \frac{2 q^{2}}{3}+O(q) \quad(q \rightarrow \infty)
$$

Using the de Caen-Selberg inequality we can improve (27) substantially as follows, which shows some difference between the two-dimensional Kakeya sets and Nikodym sets.

Theorem 5.2. Any Nikodym set $B \subset \mathbb{F}_{q}^{2}$ satisfies

$$
|B| \geqslant q^{2}-q^{3 / 2}-q,
$$

where $\mathbb{F}_{q}$ denotes a finite field of $q$ elements.

Proof. Let $s=\left|B^{c}\right|$. By the de Caen-Selberg inequality (4),

$$
q^{2}-s=|B| \geqslant\left|\bigcup_{z \in B^{c}} L_{z} \backslash\{z\}\right| \geqslant \sum_{i=1}^{s} \frac{(q-1)^{2}}{(q-1)+s-1}=\frac{s(q-1)^{2}}{s+q-2} .
$$


Equivalently,

$$
s^{2}-(q+1) s-q^{2}(q-2) \leqslant 0 .
$$

Hence

$$
|B|=q^{2}-s \geqslant q^{2}-\frac{q+1+\sqrt{(q+1)^{2}+4 q^{2}(q-2)}}{2} \geqslant q^{2}-q^{3 / 2}-q .
$$

We thank a referee for many valuable suggestions leading to the clear presentation of the paper.

\section{References}

[1] E. Bombieri, A note on the large sieve. Acta Arith. 18 (1971) 401-404.

[2] D. de Caen, A lower bound on the probability of a union. Discrete Math. 169 (1997) $217-220$.

[3] K. L. Chung, P. Erdős, On the application of the Borel-Cantelli lemma. Trans. Amer. Math. Soc. 72 (1952) 179-186.

[4] Z. Dvir, On the size of Kakeya sets in finite fields. To appear in J. Amer. Math. Soc.

[5] J. S. Ellenberg, R. Oberlin, T. Tao, The Kakeya set and maximal conjectures for algebraic varieties over finite fields. Preprint.

[6] P. Erdös, A. Rényi, On Cantor's series with convergent $\sum 1 / q_{n}$. Ann. Univ. Sci. Budapest. Eőtvős Sect. Math. 2 (1959) 93-109.

[7] X. W. C. Faber, On the finite field Kakeya problem in two dimensions, J. Number Theory 124 (2007) 248-257.

[8] C. Feng, L. Li, J. Shen, On the Borel-Cantelli lemma and its generalization. Comptes Rendus Mathematique 347 (2009) 1313-1316.

[9] S. Gallot, A bound for the maximum of a number of random variables. J. Appl. Prob. 3 (1966) 556-558.

[10] H. Heilbronn, On the averages of some arithmetical functions of two variables. Mathematika 5 (1958) 1-7.

[11] E. G. Kounias, Bounds for the probability of a union, with applications. Ann. Math. Statist. 39 (1968) 2154-2158.

[12] L. Li, On the size of Nikodym sets in finite fields. Preprint.

[13] J. E. Pečariv́, On some classical inequalities in unitary spaces. Mat. Bilten 42 (1992) 63-72.

[14] A. Rényi, Probability Theory. North-Holland Series in Applied Mathematics and Mechanics, Vol. 10. North-Holland Publishing Co., Amsterdam-London, 1970; German version 1962, French version 1966, new Hungarian edition 1965. 
[15] K. M. Rogers, The finite field Kakeya problem, Amer. Math. Monthly 108 (2000) $756-759$.

[16] S. Saraf, M. Sudan, Improved lower bound on the size of Kakeya sets over finite fields. Preprint.

[17] T. Wolff, Recent work connectecd with the Kakeya problem. Prospects in Mathematics (Princeton, NJ, 1996), Amer. Math. Soc. (1999) 129-162. 